\title{
Linx
}

Revue des linguistes de l'université Paris X Nanterre

$53 \mid 2005$

Le semi-figement

\section{À propos de certaines locutions en français}

\section{Françoise Dubois-Charlier}

\section{(2) OpenEdition \\ Journals}

Édition électronique

URL : http://journals.openedition.org/linx/259

DOI : $10.4000 /$ linx.259

ISSN : 2118-9692

\section{Éditeur}

Presses universitaires de Paris Nanterre

\section{Édition imprimée}

Date de publication : 1 décembre 2005

Pagination : $55-70$

ISSN : 0246-8743

\section{Référence électronique}

Françoise Dubois-Charlier, «À propos de certaines locutions en français », Linx [En ligne], 53 | 2005

mis en ligne le 11 février 2011, consulté le 01 mai 2019. URL : http://journals.openedition.org/linx/259 ; DOI : 10.4000/linx.259 


\title{
À propos de certaines locutions en français
}

\author{
FrançEise DubEis-Charlier \\ Université de PrEvence
}

La présente étude s'appuie sur le corpus (important, mais évidemment nonexhaustif) des locutions réunies dans Locutions en français (Jean Dubois et F. DuboisCharlier 2004), analyse syntaxique qui avait pour but et fil conducteur de montrer que les locutions se coulent dans un nombre relativement restreint de structures syntaxiques ordinaires ${ }^{1}$.

Une définition courante du terme locution est "groupe de mots figé constituant une unité du point de vue du sens » (Petit Larousse).

Nous dirons quelques mots ici sur le caractère «figé » des éléments entrant dans une locution, c'est-à-dire sur les degrés de figement, - mais peu de choses sur "l'unité du point de vue du sens », pour éviter un développement théorique sur ce que peut signifier cette expression : à un extrême, elle ne signifie pas grand'chose parce que tout constituant syntaxique, par définition, constitue une unité de sens; à l'autre extrême, elle peut signifier que le sens de la locution n'est pas déductible du sens de ses composants, ce qui n'est pas notre position. Toutes les locutions répertoriées dans le livre Les locutions sont analysables en éléments qui contribuent au sens de l'ensemble puisque l'idée de ce livre est de montrer que les locutions peuvent s'analyser en structures syntaxiques régulières.

\footnotetext{
${ }^{1}$ Les Verbes français (1994) et Locutions en français sont constitués sur les mêmes principes et reliés, dans les deux cas, à un dictionnaire électronique.
} 
Françoise Dubois-Charlier

Ce qui retiendra notre attention ici est, d'une part, ce qu'on entend, syntaxiquement, par "figé », et d'autre part, les cheminements (les raisonnements/ calculs) qui permettent de passer du sens 'propre' au sens 'figuré'.

Pour l'aspect locutionnel, nous retiendrons comme spécifique la propriété qu'ont les locutions, simultanément, de s'inscrire dans des schémas syntaxiques réguliers/classiques, et cependant de présenter divers phénomènes de particularité, de « décalage » : elles sont dans une structure syntaxique, mais légèrement à côté.

Voici quelques exemples de ce que nous entendons par « décalage ».

- la construction $\mathrm{V}+\mathrm{GN}$ est de schéma classique, on parle de "verbe transitif direct», dont le GN est le « complément d'objet direct ». Mais dans la plupart des locutions, on trouve un décalage par rapport à un vrai GV transitif comme lire Le Monde.

- d'une part, le COD n'est généralement pas passivable ; c'est un fait bien connu pour avoir et autres "middle verbs", mais qui s'étend ici à toutes sortes de verbes (dans une version adaptée du passif, où des possessifs d'identification sont nécessaires): Il a perdu la tête, *sa tête a été perdue; Paul a mis son poing dans la gueule de Max, *son poing a été mis dans la gueule de Max; Ses cris me fendaient l'âme, *Mon âme était fendue par ses cris.

- d'autre part, et ceci explique cela, l'objet n'étant pas un vrai COD, le GV est équivalent à un $\mathrm{GV}$ intransitif, souvent de type attributif ${ }^{2}$ : Il a la tête bien faite $=$ il est intelligent, Il a un poil dans la main = il est paresseux, il ne quitte pas ses pantoufles = il est casanier, il roule les mécaniques $=$ il est prétentieux, etc., ou représentant une action qui relève d'un verbe intransitif : il a un cheveu sur la langue $=$ il zozote, il a pris le mors aux dents $=$ il s'est énervé, il rendait tripes et boyaux $=$ il vomissait, il fait le joli cour $=$ il badine, etc.

- enfin, quand l'apparent COD est suivi d'un GP variable en $a ̀$, en, de, sur, comme dans casser les pieds à $G N$, mettre du plomb dans la cervelle à $G N$, secouer le paletot à GN, c'est non pas le complément direct mais le GN du GP qui, sémantiquement, est l'objet : casser les pieds à Paul $=$ ennuyer Paul, mettre du plomb dans la cervelle à Paul $=$ raisonner Paul, taper dans l'ail de Paul = impressionner Paul, etc.

- Un autre exemple concerne le jeu décalé sur le déterminant du nom, son nombre, ses modificateurs :

Paul a l'cil (quel œil défini ? pourquoi un seul ?)

copains comme les deux doigts d'une main (les 3 autres doigts ne sont pas copains?) mettre à jour vs mettre à poil (un seul poil ?)

Paul a du ventre (il n'est pas éventré ?)

etc.

\footnotetext{
${ }^{2}$ Un très grand nombre de $\mathrm{GV}=\mathrm{V}+\mathrm{GN}$ ont un correspondant sémantique de type être + Attribut ou $\mathrm{V}$ intransitif : Il mange beaucoup de pâtes $=$ il est friand de ..., Il lit trois romans par semaine $=$ il est grand lecteur de..., (verbe de type have) Il possède une fortune = il est riche. L'équivalence qui saute aux yeux pour les locutions demanderait un développement à part entière qui n'a pas sa place ici.
} 
À propos de certaines locutions en français

\title{
1. Les degrés de figement
}

À la lecture du corpus, il saute aux yeux que le figement dans les locutions connaît des degrés très variables, allant de l'impossibilité de modifier un quelconque élément de la locution à des possibilités de commutation ou de variation assez étendues et diverses ; dans ce cas, ce qui est figé, c'est le schéma de construction.

\subsection{Quelques exemples de lEcutiEns dans lesquelles En ne peut rien changer}

\author{
être sur la corde raide \\ marcher sur le fil du rasoir \\ ne pas laisser de trêve à qqn \\ laver son linge sale en famille \\ avoir une main de fer dans un gant de velours \\ mettre tous ses oeufs dans le même panier \\ avoir les bras en croix \\ être pieds et poings liés
}

NB. Nous ne comptons pas comme variation le remplacement par un autre possessif du son/sa/ses qui figure dans la locution à l'infinitif; ce déterminant est un possessif réfléchi qui s'accorde régulièrement avec le sujet - il se trouve que le français a la même forme pour le réfléchi 'indéfini' et le non-réfléchi de 3 ème personne (j'ai conduit sa voiture), mais c'est comme 'un hasard': l'anglais a une forme spéciale pour ce déterminant-là, à savoir one's, utilisée avec l'infinitif (to bear one's cross, to know one's duty), pour signaler que le possessif est à accorder en genre, personne et nombre avec le sujet. Ainsi l'accord du réfléchi est sur le même plan que les variations du verbe dans la conjugaison.

Il n'en serait pas de même pour un emploi humoristico-pédant comme Elle n'hésite pas à laver mon linge sale en famille - mais nous reviendrons sur ces 'déviations'.

Ce qu'on veut dire par $«$ strictement indissociable $=$ auquel on ne peut rien changer » peut être illustré :

1. par la comparaison avec des phrases voisines, qu'on pourrait croire, justement, relever de la commutation. Par exemple

a) Sa maladie ne laisse pas de trêve à Paul

b) Sa maladie ne laisse pas de répit à Paul

Pour b), on peut mettre au positif (La maladie lui a laissé un peu de répit, lui a laissé un répit de quelques semaines), ajouter ou changer le quantifieur (La maladie ne lui laisse aucun répit), ou procéder à une dislocation (Mais, de répit, la maladie ne lui en laisse aucun), ou à un questionnement ponctuel (Quel répit peut-on espérer que la maladie lui laissera?). Toutes modifications et reformulations qui paraissent inacceptables avec ne pas laisser de trêve (*Sa maladie lui laisse un peu de trêve, *Mais quelle trêve sa maladie lui laisse-t-elle?, etc.).

2. par le caractère ostensiblement farceur/humoristique, jouant sur les mots, de la locution avec un terme changé : soit la locution faire qqch en deux coups de cuillère à pot; 
il est loisible à un locuteur particulier de dire Elle l'a fait en 3 ou 4 coups de cuillère à pot, mais cette déformation de la locution est délibérée et doit être perçue comme telle, avec son sens "c'était moins rapide/facile qu'elle ne le croyait».

De même pour la déformation connue avoir une main de velours dans un gant de fer, par laquelle le locuteur signifie « vous connaissez cette locution qui fait référence à des gens très fermes/sévères sous des apparences souriantes/douces - eh bien moi je suis le contraire».

Sans parler des plaisanteries de type mettre tous ses cufs dans la même omelette, laver ses strings sales en famille, c'est la croix et la tanière - et les éculés je ne le connais ni des lèvres ni des dents, vieux comme mes robes et autres fort comme un bar-tabac.

3. par l'impossibilité de changer, non seulement un mot (*marcher sur la corde $\underline{\text { tendue }}$, , mais même un déterminant, ou un genre, ou un nombre:

*marcher sur un fil de rasoir, *marcher sur le fil d'un rasoir, *ces gens-là marchent sur des cordes raides, *ils mettent tous leurs aufs dans les mêmes paniers, etc.

À ce niveau, il faudrait quand même faire une distinction dans ces locutions dites totalement figées, due à l'intervention des quantifieurs, catégorie dont on sait qu'elle joue le rôle de trouble-fête dans bien des affirmations (voir le fameux couple Beaucoup de flèches n'ont pas atteint la cible et La cible n'a pas été atteinte par beaucoup de flèches, qui vers 1970 a déstabilisé la transformation passive - et toute la théorie standard de Chomsky).

Certaines locutions refusent obstinément toute espèce de quantifieur :

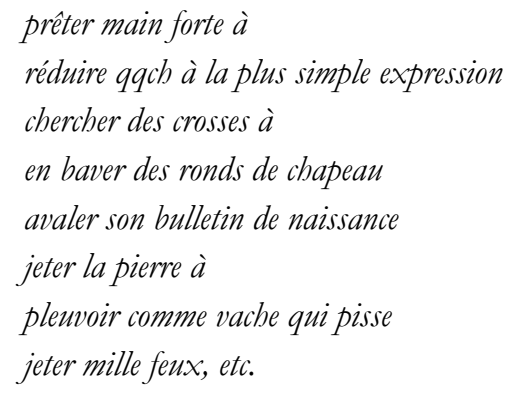

Comme les exemples le montrent, il y a divers types d'explication à ce rejet du quantifieur : pour certaines locutions, il s'agit d'une construction historique, prise telle quelle (avec son absence d'article par exemple) ; pour d'autres, c'est parce qu'il y a déjà un quantificateur (mille, tous); pour d'autres, c'est au contraire parce qu'un quantificateur n'aurait pas de sens (on n'a qu'un bulletin de naissance, une seule expression est la plus simple, ), etc.

D'autres au contraire, bien qu'apparemment très figées, admettent l'intervention d'un quantificateur, sans, me semble-t-il, qu'on ait le sentiment de la facétie :

avoir tous les atouts en main: Il a certains atouts / pas mal d'atouts en main

brûler ses vaisseaux: Il a brûlé l'essentiel/ le gros de ses vaisseaux

faire une fausse sortie: Il a fait deux ou trois fausses sorties, et il est enfin parti 
À propos de certaines locutions en français

\title{
1.2. Quelques exemples de lEcutiEns Eù En peut faire varier genre, nEmbre, déterminants, mEdificateurs
}

\author{
faire le mort, la morte \\ faire le petit fou, la petite folle, les petits fous \\ le projet a fait floc, a fait un floc retentissant \\ faire les 100 coups, les 400 coups (mais pas les 200 ou 300) \\ faire du pétard, faire beaucoup de pétard, faire un pétard d'enfer \\ piquer un petit/gros roupillon
}

- Les accords en genre et nombre sont possibles dans certaines locutions qui font intervenir l'idée d'attribut du sujet, sous une forme ou sous une autre (ne pas être un petit saint, avec féminin et pluriel). À noter cependant que le genre et le nombre peuvent être dissociés : ainsi on dit Il faisait le mort, Elle faisait la morte, mais pas * Les comparses faisaient les morts, on retombe plutôt sur le singulier Les comparses faisaient le mort. Inversement, Ce garçon n'est pas un ange, Ces enfants ne sont pas des anges, mais pas $*$ Marie $n^{\prime} e s t$ pas une ange (la question du sexe des anges est très débattue, mais le genre du mot, lui, ne fait aucun problème).

Souvent le nombre est fixe, avec une certaine logique :

$$
\begin{aligned}
& \text { nous l'entendions crier à fendre l'âme (de chacun) } \\
& \text { il leur a cassé la figure (à chacun) } \\
& \text { elle m'a cassé les pieds (j'en ai deux) } \\
& \text { ils ont la gueule enfarinée (chacun une) } \\
& \text { tirer à la courte paille (une seule est courte) } \\
& \text { tirer à boulets ronges (plusieurs boulets) } \\
& \text { se regarder en cbiens de faïence (réciproque, } 2 \text { chiens) }
\end{aligned}
$$

et naturellement il est fixe quand il est exprimé par un cardinal :

$$
\begin{aligned}
& \text { courir deux lièvres à la fois } \\
& \text { faire d'une pierre deux coups } \\
& \text { se mettre en quatre } \\
& \text { se saigner aux quatre veines }
\end{aligned}
$$

(le changement fait plaisantin : se mettre en 8 ou 16, faire d'une pierre 3 coups - tout comme le rajout d'un numéral là où il est redondant : je l'ai supplié à deux genoux).

Mais la logique est loin d'être loin d'être toujours au rendez-vous :

taper dans l'ail de qqn (on en a deux - nous reviendrons sur les parties du corps) je lui en toucherai un mot (il en faut souvent plus d'un)

tomber dans les filets de qqn (on ne tombe que dans un seul)

connaitre qqch comme sa poche (les hommes ont toujours trente-six poches)

sonder les reins et les cours (on n'a qu'un cœur - ça fait plusieurs au total, mais ceci serait vrai de faire suer le burnous)

on se lève avec/comme les poules mais on est rouge comme un coquelicot et on vous fait prendre des vessies pour des lanternes (même quand il ne s'agit que d'une méprise), etc. 
- Les modificateurs qu'on peut ajouter semblent être surtout de type quantitatif, emphatique, augmentatif ou diminutif: un floc retentissant, passer un savon de tous les diables, sombrer dans le désespoir le plus noir, dans un profond désespoir. Sans parler des « hedges » et des intensifs : faire un four, faire comme un four, faire une manière de four, faire comme qui dirait un four, faire un vache de four, etc.

\subsection{Une certaine variabilité à l'intérieur d'un schéma syntaxique}

À titre d'exemple, prenons un schéma très représenté dans le corpus, $V$ spéc + GN variable + Attribut/Manière spéc: le verbe et le second complément sont fixes, le COD est plus ou moins libre.

\section{Le GN COD est variable à l'intérieur d'un paradigme}

a) Le paradigme est lexical, les $\mathbf{N}$ eux-mêmes doivent appartenir à une série lexicale.

Chauffer qqn à blanc implique généralement "pour une occasion donnée, de type prestation, épreuve, spectacle»; le GN humain COD est limité à une petite liste de termes désignant des individus se préparant à donner une prestation. Ces termes peuvent être énumérés, la liste est finie :

$$
\begin{aligned}
& \text { Les organisateurs avaient chauffé à blanc } \\
& \text { - la salle, le public, l'auditoire } \\
& \text { Le candidat était chauffé à blanc }
\end{aligned}
$$

Quand le GN contient un autre N, ou un nom propre, un pronom, le terme lexical du paradigme est normalement présent dans le contexte :

J'avais préparé mon intervention à fond, j'étais chauffé à blanc

Paul les avait chauffés à blanc (contexte précédent: ils se préparaient à une prestation).

b) Le paradigme est sémantique, il s'agit de la référence des mots (pas des $\mathbf{N}$ eux-mêmes). Ainsi dans :

prendre qqch au pied de la lettre

le GN COD peut être représenté par une série assez variable de mots et de constructions, mais toutes ces expressions doivent référer à du texte ou du discours. Les unités lexicales ne peuvent pas vraiment être énumérées, notamment parce qu'on trouve souvent des relatives :

$$
\begin{aligned}
& \text { Il a pris au pied la lettre } \quad \text { - ces instructions, l'article du journal } \\
&- \text { ce que tu as écrit, tout ce qu'il entendait } \\
& \text { Il prend tonjours an pied de la lettre tout ce qu'on lui dit, tout ce qu'il lit. }
\end{aligned}
$$

\section{c) La contrainte est dans l'extralinguistique}

se mettre qqn à dos 
peut illustrer un autre type de contrainte qui n'apparait pas dans les mots constituant le COD : celui-ci peut contenir n'importe quel nom, propre ou commun, désignant un humain ou groupe humain, mais l'expression n'est appropriée que si, dans la situation, les référents du sujet et de l'objet ont entre eux une relation humaine ou sociale. Celleci peut apparaitre dans les termes du COD :

Il s'est mis à dos ses collègues, ses clients, ses enfants, ses voisins

mais ce n'est pas nécessaire. C'est le contexte situationnel qui compte :

Il se met tout le monde à dos

Il s'est mis à dos mes amis, mon frère, Pierre et Paul

La contrainte sur cette relation situationnelle implicite apparaît dans le caractère incongru d'une phrase comme :

\section{En achetant ce pullover / En confectionnant cette tarte, je me suis mis G. Bush à dos}

En m'entendant dire cette phrase (moi, FDC), un inconnu serait obligé de forcer une interprétation du genre 'son ami Bush lui avait interdit d'acheter des pulls ou de faire des tartes' - quant aux gens qui me connaissent, et savent que je n'ai aucune espèce de relation avec Bush, ils devraient conclure que mon discours relève d'un épisode délirant.

\section{Le GN COD est tEtalement variable}

NB. On ne prend pas en compte la contrainte tout à fait générale, pour toute phrase, concernant la possibilité de la relation prédicative (compatibilité entre le sujet et le prédicat, circonstances possibles, etc.). Si on la prenait en compte, aucun constituant ne pourrait être dit 'libre'.

avoir qqn/ qqch à l'ail (au sens de 'surveiller attentivement')

le GN COD peut référer à toute espèce d'objet ou individu qui figure dans l'univers du sujet :

Il a à l'ail - les fluctuations de la Bourse, son régime, les appartements en vente à Aix, - l'évolution du parti socialiste, la météo pour le week-end, etc.

Il en est de même sans doute pour :

passer GN à l'as

= n'importe quel fait ou existence/bien-être/désir de n'importe qui de son univers

prendre qqch/qqn au sérieux

= n'importe quel événement, individu, objet, fait, de son univers 


\section{2. Étude plus détaillée d'un sous-corpus : les locutions impliquant les parties du corps}

Pour mieux cerner les phénomènes, portons maintenant notre attention sur un sous-corpus, les locutions impliquant des mots désignant les parties du corps. Pourquoi ce choix ? D'une part, par facilité/banalité : c'est une idée évidente, qui vient tout de suite à l'esprit. D'autre part, parce que ce domaine est très étoffé : les locutions impliquant les parties du corps représentent presque $20 \%$ de notre corpus total (1 400/7 500). J'examinerai, sur ce sous-corpus, deux rubriques :

- la constitution de ces locutions ;

- les sources de l'emploi figuré.

\section{1. Étude syntaxique du sEus-cErpus.}

Un coup d'œil superficiel sur le sous-corpus permet de relever par exemple :

tenir à bout de bras (compl. de nom) ${ }^{3}$, accueillir à bras ouverts (dans GP de manière),

les bras m'en tombent (sujet),

il joue petits bras (manière sans prép.),

ça m'a coupé bras et jambes (COD),

il a le bras long (COD + attribut), etc.

À cette énumération, très partielle, on pourrait penser que, dans ces locutions, les constructions sont multiples, variables; c'est l'idée qu'évoque pour certaines personnes le concept d'«expression figée », set phrase: «qui n'obéit pas aux règles habituelles/normales de formation ».

Rien n'est plus faux, naturellement. Comme dit en introduction, ce qui a intéressé Jean Dubois dans son étude des locutions, c'est de mettre au jour leurs patterns systématiques de construction. Et je vais développer cette idée sur ce corpus particulier.

Les quelque 1400 locutions notées (sous-ensemble abrégé ci-dessous en 'corpus') s'analysent en un petit nombre de schémas syntaxiques.

\section{A. Le schéma $V$ transitif suivi de sEn Eu ses cEmpléments}

NB. Nous gardons ici l'étiquette conventionnelle 'transitif', bien que, comme dit plus haut, il n'y ait pas de passif, etc.

Quatre verbes supports représentent à eux seuls le tiers du corpus : avoir (310), faire (80), mettre (30), prendre (20), total 440/1 400. Si l'on ajoute quelques autres verbes,

\footnotetext{
${ }^{3}$ La parenthèse indique le statut du $\mathrm{N}$ de partie du corps.
} 
donner (12), ouvirif fermer (12), casser (10), perdre (9), porter (8), tirer (8), jeter (8), serrer (6), on obtient presque $40 \%$ du corpus (513/1 400).

On remarquera au passage que seuls un ou deux de ces verbes font spontanément penser à des parties du corps : pour casser, serrer, pas de problème, mais d'autres sont à première vue surprenants : perdre une partie du corps ?, la jeter ? - tout trouvera sa place finalement. Naturellement, étant donné le V et son COD, le sujet est massivement un animé humain.

Les schémas pour le $\mathrm{V}$ transitif se distinguent ainsi :

\section{COD tEut seul}

- minimal : avoir de l'estomac, de la gueule, du ccur, des couilles, l'cill, la dent, toute sa tête - avec modificateur épithète ou complément de nom

avoir bon ceur, mauvais esprit, la grosse tête; avoir une tête à claques, du sang de navet, un cour d'artichaut

a) Dans le schéma à COD minimal, la partie du corps est donc le nom tête de ce GN, accompagné seulement d'un déterminant. Il est intéressant alors d'examiner ce déterminant. Bien logiquement, l'indéfini un/une n'est pas représenté : des phrases de type j'ai un estomac, avec le sens propre pour la partie du corps, étant totalement vides d'information, sont contraires aux maximes de la conversation de Grice («be relevant») et au principe de coopération. Si un témoin prié de décrire le suspect qu'il a vu répondait: il avait un visage, deux yeux, un nez et une bouche, l'inspecteur le prendrait mal. Ce qu'on trouve essentiellement est :

1. le partitif/quantitatif : on a ici un autre sens du mot désignant la partie du corps, par ex. pour ccur, passage de l'organe physique au lieu supposé d'émotions ou sentiments, c'est-à-dire des indénombrables, ce qui se traduit par le partitif $d e / d u / d e$ la (au sens de 'beaucoup de') : il a du cour = il a de la bonté, il a de l'estomac = il a de l'aplomb, de la hardiesse, etc.

2. le défini, où le passage au sens figuré peut se traduire par une déviation du nombre: il a l'xil (alors qu'il en a deux comme tout le monde), j'ai la dent (heureusement j'en ai plus d'une), et inversement il a les foies (alors qu'il n'en a qu'un).

Dans d'autres cas de défini, la partie du corps est représentée par un mot emprunté (avoir les crocs) ou un mot qui fait image/symbole (avoir la trique, le bambou), sémantiquement équivalent à 'avoir $\mathrm{N}$ attribut' = avoir la verge raide.

3. Restent quelques locutions où l'article est bien celui qui convient pour la réalité corporelle, comme dans avoir des couilles (effectivement, elles vont par deux), et le sens figuré provient justement du fait qu'on ne peut attribuer la possession concrète de cette paire ni à un homme (tautologique), ni à une femme (contre-factuel). On retombe sur le quantitatif implicite 'avoir de grosses couilles', stéréotype de virilité, stéréotype d'audace, de décision, etc.

b) Dans le schéma à COD contenant un modificateur, pour l'adjectif antéposé on ne trouve guère que bon/mauvais, grand/petit, sans article [sauf avec tête] : avoir une 
petite tête, la grosse tête, faire sa manvaise tête, et quelques autres : faire triste mine, faire pâle figure. Plus intéressante est la série où la partie du corps est accompagnée d'un complément de nom : avoir une tête de mule, une âme de valet.

Cette série présente une grande homogénéité de forme : article un/une pour la partie du corps, le complément de nom est sans article. Mais elle peut se subdiviser en plusieurs types selon le rôle du GP, qu'on peut mettre en évidence par des gloses :

il a/c'est un cour d'artichaut = «son cœur est comme un artichaut», il en donne un bout à tout le monde, comme on effeuille un artichaut

il a un estomac d'autruche = "son estomac est comme celui d'une autruche», susceptible de digérer n'importe quoi

Ces comparaisons sont essentiellement avec des animaux ou des végétaux (yeux de lynx, tête de cochon, peau de pêche) et elles s'opposent en cela à des locutions comme :

il est arrivé avec une figure d'enterrement : non pas «*sa figure était comme un enterrement », mais bien "était comme celle qu'on a pendant un enterrement» il a une tête à claques/à gifles : là, c'est « sa tête est telle qu'on a envie de lui donner des claques »

Dans ces deux derniers cas, le GP ne désigne pas une autre entité comparable, mais un événement, une circonstance ou une action (enterrement, claque).

\section{COD + attribut de l'Ebjet}

Typiquement le sujet est un humain et le COD est une partie du corps.

a) L'attribut est un adjectif (ou participe passé adjectivé) : avoir l'échine souple, la conscience nette, le gosier sec, la tripe républicaine, les reins solides.

Cette structure-là est une des plus exploitée dans le corpus, et toutes les locutions présentent une très grande homogénéité de forme; l'article est systématiquement le défini, le/la/les, et le nombre est le plus souvent respecté : avoir la langue bien pendue, $\underline{\text { le }}$ nez creux, les jambes coupées, les yeux battus, les chevilles enflées. Avec cependant quelques déviations de nombre (par rapport au monde référentiel) : avoir l'oreille musicale, la cuisse légère, $\underline{\text { la }}$ main leste, $\underline{\text { la dent dure. }}$

b) L'attribut est un groupe prépositionnel : avoir les doigts de pied en éventail, les yeux en boules de loto, la bouche en cul de poule, les jambes en pâté de foie, un visage en lame de couteau.

Là encore, grande homogénéité de forme : l'article est défini le/la/les (sauf deux exceptions) pour la partie du corps; le GP attribut, décrivant une forme ou une taille/disposition, est avec la préposition en et sans article (avec un nom qui peut être dénombrable ou non) : avoir le nez en pied de marmite. 

systématique $^{4}$ :

Ces locutions se répartissent en deux groupes ayant chacun sa glose - «Son N est (comme) tel objet»: il a le nez en trompette, en pied de marmite $=$ «son nez est comme, a la même forme que une trompette, un pied de marmite »

il a les yeux en amande = «ses yeux sont comme des amandes » (ont la même forme)

il avait les doigts de pied en bouquet $=$ « ses doigts de pied étaient écartés comme les tiges des fleurs formant un bouquet»

il avait les bras en croix = "ses bras étaient avec son corps comme les deux branches d'une croix » (perpendiculaires), etc.

- «Son N est en telle forme/direction »:

il a les côtes en long = "ses côtes sont allongées » (pas serrées par/pour l'action) elle a les joues en feu = "ses joues sont rouges, comme sous l'action du feu » il a la dalle en pente = "sa dalle [gosier] est en pente » (l'alcool y descend très facilement), etc.

[Exceptions, pour l'article: avoir un visage en lame de couteau, et, pour la préposition : avoir les nerfs à toute épreuve]

\section{3. $C O D+G P$ (essentiellement de lieu)}

avoir un poids sur l'estomac, un poil dans la main, un cheveu sur la langue, le diable au corps, un pied dans la tombe, du jus de navet dans les veines, la tête près du bonnet, de la merde dans les yeux.

C'est une grande série, à peu près aussi représentée que avoir le $N$ Adj. Là, on trouve tous les articles, défini avoir le cour sur la main, le nez dans son caca, les pieds sur terre, indéfini un pied dans la tombe, une main de fer dans un gant de velours, partitif du sang sur les mains, du poil au cul. Et pour le GP, on trouve toute la gamme des prépositions de lieu : dans, sur, à, etc.

Il faudrait sans doute subdiviser le schéma en trois sous-catégories selon la place et la fonction du mot désignant la partie du corps.

a) la partie du corps est le COD : avoir le doigt sur la détente, la main au portefenille, le dos au mur

b) la partie du corps est dans le GP :

avoir les armes à la main, un verre dans le nez, un chat dans la gorge, le couteau sous la gorge, tous les atouts en main, le feu aux fesses

\footnotetext{
${ }^{4}$ Il s'agit de gloses, 'artificielles' en somme, celles-ci ne s'emploieraient pas vraiment à la place de la locution, et, si elles étaient employées, alors l'aspect locutionnel et le sens figuré disparaîtraient.
} 
c) il y a deux parties du corps (en COD et en GP) : avoir l'estomac dans les talons, le ccour sur la main, la tête sur les épaules, du cour au ventre

Enfin il faudrait ajouter deux autres petites sous-catégories :

- avec COD sans article (parfois analysé comme adverbial) : avoir mal aux chevenx, aux chevilles, avoir chand an cour, aux fesses, ne pas avoir froid aux yeux

- GN libre introduit par de et précédé d'un en anticipatoire : en avoir plein la bouche, le cul, le dos, les pattes de GN en avoir par dessus la tête, ras le bol de GN

\section{Le COD est variable, la partie du cErps est dans le GP}

avoir GN à caur, dans la peau, dans le nez, dans le pif avoir $G N$ en tête, bien en main, sur le bout de la langue, etc.

\section{Le GN du GP est variable, la partie du cErps est COD (petite série)}

avoir l'oreille de GN, la haute main sur GN, une dent contre GN, etc.

\section{B. Un autre schéma très prEductif est la structure PrépEsitiEn $+G N$}

Les locutions relevant de cette structure représentent environ un tiers du corpus. Exemples : de bon cour, jusqu'au cou, sur le flanc.

Pour l'analyse en schémas syntaxiques, ces locutions posent un problème évident (et le flottement peut se voir dans l'index du livre Les locutions!). Quand le GP accompagne toujours le même verbe, alors il s'agit d'une locution verbale (ex : courir sur le baricot); quand le GP peut accompagner des verbes bien différents, alors la locution sera classée prépositionnelle (ex : de bon cour - rire, accepter, prêter, obéir, se mettre au travail, etc.). Mais il y a tous les intermédiaires, avec deux ou trois verbes préférentiels, avec des verbes synonymes, etc. (ex : sous le coude $[d e]$ ne va qu'avec quelques verbes liés entre eux, mais on hésite quand même à le figer en garder sous le coude pour ne pas exclure les phrases avec avoir, être, se mettre, rester, etc.).

Il serait trop long d'entrer dans le détail de ces constructions en Prép $+G N$, on se contentera de signaler :

1. qu'elles exploitent toutes les possibilités régulièrement permises par la structure :

- toute la gamme des prépositions françaises (y compris l'effacement de la préposition, comme dans la tête lourde ou la mort dans l'âme);

- toutes les possibilités de constitution du GN : avec ou sans déterminant (à la barbe de, à deux doigts de, à vue d'ail); avec ou sans modificateur adjectival (en pleine figure, en culottes courtes, sur les genoux); avec ou sans modificateur complément de nom (dans un coin de son cour, sur le bout des lèvres), etc. 
2. qu'elles illustrent le jeu sur le déterminant du nom, son nombre, certains de ses modificateurs : d'une oreille favorable, sur un bon pied, de sa plus belle main, à deux visages, mettre le couteau sur ou sous la gorge, etc.

\section{2. Étude sémantique du sous-corpus : les sources $d u$ figuré}

A. Dans un survol général du corpus total des locutions, on peut distinguer plusieurs sources pour le passage au figuré. Par exemple, et à très grands traits :

1. Un nom donné est à prendre au sens de «n'importe quel $\mathrm{N} »$ : appeler un chat un chat = «appeler une chose quelconque par son nom» (dans la locution parallèle en anglais, c'est d'une bêche qu'il s'agit).

2. Le nom représente une propriété stéréotypée de l'objet: Des clous! nêfles! haricots! = les clous, les nèfles, les haricots sont des choses peu appréciées, ne valent pas grand'chose; de même dans ça vaut trois sous espagnols et travailler pour des prunes.

3. Conséquence stéréotypée imaginaire d'une propriété du sujet : elle est belle à faire damner un saint.

On notera dans ce type d'expression deux syntaxes : Il est bête à manger du foin (c'est lui qui pourrait en manger) $\neq I l$ est bête à pleurer (c'est nous qui pourrions pleurer).

4. Comparaison avec une propriété stéréotypée du comparant: monté comme un taureau, maigre comme un hareng saur.

À noter que ces stéréotypes sont très variés, souvent personnels, et se prêtent à de multiples exploitations : à partir de comme une poule qui a trouvé un cure-dents, l'idée de «dents » entraine le raffinement qui a trouvé un peigne, puis le peigne apparait comme étant un objet quelconque et on a qui a trowvé une cuillère, un fer à repasser, etc.

À noter aussi l'effet rétrospectif de certaines de ces comparaisons pour qui n'en connait pas l'origine (c'est-à-dire à peu près tout le monde) : con comme un balai (un balai est plus con qu'une serpillière?), soûl comme une grive (la grive est poivrote ?), etc.

5. L'image pure et simple :

avoir du vent dans les voiles (ne pas aller très droit --> être un peu ivre)

cet épisode, pour moi, c'est le trou noir (lieu où rien n'est visible --> je n'en ai aucun souvenir)

brouiller les cartes, briser la glace, démarrer sur les chapeaux de roue

Le figuré est dû au fait que l'objet n'est pas là : on n'a pas de voile, on n'a pas de cartes, de glace, etc., à la différence du cas suivant. 
Françoise Dubois-Charlier

6. Extension du propre pour un certain individu à un autre individu : Paul est bas sur pattes (extension de la description, au propre, d'un teckel).

7. Le contenant pour le contenu ou le tout pour la partie, ou l'inverse :

Paul en a plein la bouche de ce projet (bouche = paroles)

pleurer toutes les larmes de son corps (être très triste, déçu, navré)

montrer le bout de son oreille/ nez. (sa personne, discrètement)

8. Attribution d'une propriété par la relation stéréotypée entre deux autres entités : être le jour et la nuit, être cul et chemise avec, ménager la chèvre et le chou. etc.

B. Dans le corpus, sévèrement limité, des locutions contenant des mots désignant des parties du corps, on ne trouve naturellement pas toutes les sources de passage au figuré, et certaines sont beaucoup plus exploitées que d'autres.

1. L'une des plus exploitées est la simple extension; c'est à peine figuré, simple passage du physique au mental ou à l'abstrait, le propre est possible et pourrait, dans un autre contexte, s'appliquer au même sujet:

trainer la jambe, la main dans la main, bras dessus bras dessous, sur la pointe des pieds, rejeter d'un revers de main, se compter sur les doigts de la main, donner un coup de pied au cul, faire un pied de nez à, faire venir l'eau à la bouche, se boucher les oreilles, fermer les yeux, avoir la gorge sèche, baisser les bras, se croiser les bras, etc.

2. Déplacement du propre pour un certain sujet au figuré pour un autre: rester l'arme an pied (propre pour le soldat)

avoir tous les atouts en main (propre pour le joueur de bridge)

avoir du sang sur les mains (propre pour le meurtrier)

mettre le conteau sous la gorge (propre pour l'agresseur à main armée)

prendre le mors aux dents (propre pour cheval)

revenir la queue entre les jambes (propre pour le chien - et tautologique pour l'homme)

3. Le passage au figuré est simplement dû au fait que le propre serait physiquement impossible ou contre-factuel :

şa m'a coupé bras et jambes (je les ai encore)

je suis morte de rire/faim/peur (je suis vivante)

ça m'a coûté les yeux de la tête (id)

prendre ses jambes à son cou (si on le faisait, on n'irait pas vite ni loin)

j'ai donné ma langue au chat

avoir les yeux plus grands que le ventre, etc.

4. La partie du corps est le symbole d'une activité, capacité, etc. :

bouche : siège de la parole --> discours, paroles, le dit : 
apprendre par la bouche de, être dans toutes les bouches, se dire/ répandre de bouche à oreille, en avoir plein la bouche de, garder pour la bonne bouche (la conclusion la plus intéressante d'un exposé), fermer la bouche à ou clouer le bec à (l'empêcher de s'exprimer)

yeux : organe de la perception --> jugement :

jeter de la poudre aux yeux = chercher à faire illusion, empêcher de voir/juger lucidement

ne pas avoir les yeux dans sa poche = n'avoir pas le jugement handicapé

fermer/se boucher les yeux sur = refuser d'enregistrer, de tenir compte

ne voir que par les yeux de = faire siens ses jugements

ça me sort par les yeux, par les trous de nez, par les oreilles, par tous les pores, etc., orifice pour le débordement. A noter que l'idée de « ras le bol » est très présente dans les locutions avec parties du corps, et sous diverses images : en avoir par dessus la tête, jusqu'au cou, plein les bottes/le cul, etc. bras : à côté de l'organe de l'action et la force, il y aussi le moyen d'entourer qqn, d'où le passage aux êtres mythiques :

être dans les bras de Morphée, s'endormir dans les bras du Seigneur

main : l'organe de la préhension --> tenir, posséder, dominer, maitriser :

avoir/prendre les choses en main

avoir la haute main sur

tomber aux mains de

5. Dans un certain nombre de cas, c'est un mot autre que la partie du corps qui, par son association avec la partie du corps, déclenche l'image et le passage au figuré :

avoir un cheveu/fil sur la langue (zozoter, zézayer)

avoir un bouf sur la langue (se taire)

fondre dans la bouche (à propos d'une viande)

parler entre quatre yeux (en tête à tête)

\section{Conclusion}

À l'aide d'exemples tirés de Locutions en français, on a cherché ici à :

- illustrer la gamme des degrés de figement, depuis les locutions dont tous les composants sont rigoureusement intangibles jusqu'à celles où un ou plusieurs constituants sont totalement variables ;

- montrer que les locutions se coulent dans des structures syntaxiques ordinaires et régulières, mais qu'en même temps, elles manifestent diverses particularités dans les constructions dont elles relèvent (le COD n'est pas un 
« vrai » $\mathrm{COD}$, on a un singulier là où la syntaxe demande un duel, la préposition flotte, etc.) ;

- explorer, sur un sous-corpus constitué par les locutions impliquant des noms de parties du corps, certaines des sources du passage au figuré - pour étayer l'idée que, là encore, il y a des régularités et que les phénomènes peuvent s'analyser en procédés répertoriés (ou à répertorier).

Une qualification possible pourrait être de dire que la locution représente une étape intermédiaire entre la phrase libre (propre ou métaphorique) et la phrase figée qu'est le proverbe.

\section{RÉFÉRENCES}

ANSCOMBRE, J.-C. (éd.) (2000), La parole proverbiale, Langages 139.

BerCKer, F. (1992), « Alouette, gentille alouette...Parties du corps et constructions figées », in I. Perrin (éd.) Approches énonciatives de l'énoncé complexe, Paris, BIG, pp. 119-143.

Dubois, J. ; Dubois-Charlier, F. (2004), Locutions en français, Aix en Provence, Soibud, 414 p.

Dubois, J. ; Dubois-Charlier, F. (1995), Dictionnaire électronique des locutions, [5 810 entrées], Aix en Provence, chez les auteurs.

Dubois, J.; Dubois-Charlier, F. (1994), Les Verbes français (d'après le «Dictionnaire électronique des verbes »), Paris, Larousse, 458 p.

Dubois, J. ; Dubois-Charlier, F. (1997), «Synonymie syntaxique et classification des verbes français ", Langages 128, La synonymie, A. Balibar-Mrabti (éd.), pp. 51-71.

Grice, H. P. (1975), «Logic and conversation », in P. Cole \& J.l. Morgan (eds.), Syntax and Semantics 3 : Speech Acts, New York, Academic Press., pp. 41-58.

Gross, G. (1993), «Trois applications de la notion de verbe support », L'Information grammaticale 59 , pp. $16-22$.

GROss, M. (1990), Grammaire transformationnelle du français 3 : l'adverbe, Paris, ASSTRIL.

JaCKendofF, R. S. (1969), «An Interpretative Theory of Negation », Foundations of Language 5, pp. 218-244.

Martins-Baltar, M. (éd.) (1997), La locution entre langue et usages, Paris, ENS éditions.

Simatos, I. (1997), «Expression idiomatique, expression figée, prédicat complexe », in B. Fradin et J.M. Marandin (dirs), Mots et grammaires, Paris, Didier Erudition, pp. 89-127. 